\title{
Comparative Analysis of Monitoring Methods for Vortex-induced Vibration of Multistage Pressure Reducing Valves
}

Dongtao Xu ( $\sim$ as_xudongtao@163.com )

University of Science and Technology Liaoning

Ge Chang-rong

University of Science and Technology Liaoning

Li Ying

University of Science and Technology Liaoning

Liu Yue-juan

University of Science and Technology Liaoning

\section{Research Article}

Keywords: Multistage pressure reducing valve, Vortex-induced vibration, Lift coefficient, Pressure fluctuation, Modal analysis, Computational fluid dynamics (CFD)

Posted Date: November 17th, 2021

DOI: https://doi.org/10.21203/rs.3.rs-1060582/v1

License: (c) (i) This work is licensed under a Creative Commons Attribution 4.0 International License. Read Full License 


\section{Abstract}

In this paper, a multistage pressure reducing valve is presented. The main frequency of vortex-induced vibration is evaluated by monitoring the lift coefficient during vortex shedding and the pressure fluctuation formed after vortex shedding in the flow field. By comparative analysis of two different methods, the number of vortices is relatively small at small openings. Due to the limitations of the location and quantity of monitoring points, accurately locating the most active position where pressure fluctuation occurs is difficult. Monitoring the lift coefficient is more suitable to evaluate the main frequency of vortex-induced vibration. At medium and large openings, due to the increase in the number of vortices, the superposition effect of the pressure fluctuation and the influence of the flow channel shape is more obvious. Monitoring the pressure fluctuation is more appropriate to evaluate the main frequency of vortex-induced vibration the valve. Therefore, a combination of the two methods can more accurately evaluate the vortex-induced vibration characteristics of the valve. When monitoring pressure fluctuation, the position and number of monitoring points directly affect the evaluation accuracy. The pressure fluctuations around the outlet and the multilayer sleeve are more active. It is more meaningful to monitor the pressure fluctuation at these points. The main frequency of the pressure fluctuation at these points better reflects the vortex-induced vibration characteristics of the valve.

\section{Introduction}

The regulating valve is an indispensable component in modern control systems, as it can control flow rate and stabilize pressure [1-3]. This valve is widely used in petrochemical, water conservancy, energy and other industrial sectors. With the development of science and technology, to improve the reliability of control systems, higher requirements are proposed for the design rationality of throttle components in regulating valves. To realize the functions of throttling and reducing pressure, the pressure reducing components of a valve are designed as labyrinth disc, window and porous sleeve types. Some scholars [4-5] have studied the combined structure of labyrinth discs and multistage sleeves to achieve pressure and noise reduction functions. In actual control systems with high temperature, pressure and other factors, when a fluid flows through the throttling components, vortices may form near the throttling components. This vortex shedding is periodic and has a main frequency. After vortex shedding, pressure fluctuations form and are superimposed on each other which produces pulsating shocks. When the main frequency of vortex shedding or the pressure fluctuation is equal to or close to the natural frequency of the valve, vortex-induced resonance will occur. This will cause large displacements and deformations in the valve trims, resulting in considerable vibration and noise that affects the safety of the control system.

Some scholars have researched vortex-induced vibration phenomena in valves. Wang Hai-min [6-7] calculated the natural frequencies of a triple-eccentric butterfly valve and combined the frequencies with the frequency calculation formula of the Karman vortex street to determine the conditions for no resonance. Kang Zhuang [8-9] studied the effects of hysteresis, surface roughness and other factors on the vortex-induced vibration of vertical pipes. Chizfahm A [10] studied the influence of wind speed on the lift coefficient of turbines and concluded that vortex shedding and structural oscillation were 
synchronized. Youn [11] analyzed the flow of compressed air through orifices and radial slits based on CFD and obtained pressure fluctuation curves under different flow conditions.

A multistage pressure reducing valve is presented in this paper. The pressure reducing components are shown to effectively prevent cavitation vibration in the valve caused by a sharp decrease in fluid pressure. The simulation shows that many vortices appear around the multilayer sleeve. Two different methods are used to analyze the main frequency of the vortex-induced vibration of the valve. The first method is to directly monitor the main frequency of vortex shedding by monitoring the lift coefficient, and the other method is to monitor the pressure fluctuations at points in the valve and the fluid-solid coupling surface. However, there are differences in the monitoring results of these two methods. Therefore, by analyzing the monitoring mechanism, advantages, and disadvantages of the two monitoring methods, the vortexinduced vibration characteristics of the valve can be more accurately evaluated.

\section{Structural Description Of The Multistage Pressure Reducing Valve}

The structure of the multistage pressure reducing valve is shown in Fig. 1. The valve consists of a valve body, valve seat, multilayer sleeve, valve plug, pressure cage, upper bonnet, and valve rod. The valve rod is fixed with the valve plug, and the valve rod is connected with an actuator. The valve rod is driven by the external actuator and is able to drive the valve plug to move up and down. In this manner, the orifices on the inner sleeve of the multilayer sleeve are exposed to form effective flow areas.

When a fluid flows through the multilayer sleeve, the fluid velocity increases sharply as the flow area decreases quickly, and the hydrostatic pressure of the fluid drops. If the fluid pressure suddenly drops to the saturated vapor pressure, cavitation vibration will occur [12]. If the pressure difference of each stage pressure drop is greater than the blocked flow critical pressure difference, blocked flow will occur. The multilayer sleeve can gradually reduce the pressure of the fluid in the valve to prevent cavitation vibration and block flow. The structure of the outer sleeve and the middle sleeve can be designed by assigning the same orifices. The inner sleeve not only reduces pressure but also controls the flow rate at each valve opening.

When the fluid in the valve is liquid water, the diameter of the valve is $\Phi 250 \mathrm{~mm}$, the valve plug diameter is $\$ 165 \mathrm{~mm}$, and the inlet and outlet pressures are 10 and $3 \mathrm{MPa}$, respectively. The valve is designed according to the linear flow characteristics $\left(C_{V}=360\right)$. The multilayer sleeve structure is designed as shown in Fig. 2.

\section{Flow Field Simulation Of The Multistage Pressure Reducing Valve 3.1 Establishing the simulation model.}

Before simulation, a 3D model of the valve is established, as shown in Fig. 1. The structure of the simulation model is established according to the above design parameters. The pressure at the inlet of 
the valve is $10 \mathrm{MPa}$, and the pressure at the outlet is $3 \mathrm{MPa}$. The fluid model corresponding to each opening is generated by reverse modeling. The fluid in the valve is liquid water with a temperature of $473.15 \mathrm{~K}$. To simulate the flow field of the valve, the fluid model is divided into tetrahedral and hexahedron hybrid meshes using the ANSYS meshing tool, and local refinement is used at the orifices. The grid independence is checked, and the reference value of grid independence is based on the flow rate and average flow velocity at the valve outlet at a $100 \%$ opening. The test data is shown in Table 1:

Table 1

Fluid grid independence test data

\begin{tabular}{|lll|}
\hline Number of grid cells & $\begin{array}{l}\text { Flow velocity } \\
(\mathrm{m} / \mathrm{s})\end{array}$ & $\begin{array}{l}\text { Flow rate } \\
\left(\mathrm{m}^{3} / \mathrm{h}\right)\end{array}$ \\
\hline 3951741 & 15.698 & 2772.558 \\
\hline 4263695 & 15.709 & 2773.955 \\
\hline 5177818 & 15.805 & 2789.258 \\
\hline 6361212 & 15.811 & 2788.952 \\
\hline
\end{tabular}

According to Table 1, when the number of grids increases from 5177818 to 6361212 , the flow velocity at the outlet increases by $0.038 \%$, and the flow rate decreases by $0.01 \%$. When the number of grid cells is greater than 5177818 , the change in flow velocity and flow rate at the outlet can be ignored. Further refinement of the finite element mesh will not significantly affect the simulation results. At this time, the grid independence requirements are satisfied. Figure 3 shows the fluid mesh model.

\subsection{Selection of turbulence model for transient flow field}

The RNG $k-\varepsilon$ turbulence model is used for transient flow field simulation in this paper. The RNG $k-\varepsilon$ model is based on Standard $k-\varepsilon$ and is used to simulate the transient flow field. In complex flow fields, numerical simulation is more accurate when the RNG $k-\varepsilon$ model is used but difficult to converge [13-14]. Variable parameters in the model are correlated with the fluid model, fluid flow state and corresponding space coordinate system functions. The RNG $k-\varepsilon$ model can effectively manage the turbulent flow in the piping system of the regulator valve.

\subsection{Numerical simulation of the flow field 3.3.1 Numerical simulation of the flow rate.}

The maximum stroke of the valve plug is $100 \mathrm{~mm}$. The inlet of the valve is on the left side. The flow rate at the outlet of the valve is monitored. After iterative calculations, the simulation shows that the circulation volume of the valve $(\mathrm{Cv})$ is 358.96 . The relative flow coefficient obtained by simulation is compared with the standard theoretical value, as shown in Fig. 4. 
$C$, the relative flow coefficient, is dimensionless and is the ratio of the flow rate at a certain opening to the circulation volume $(\mathrm{Cv})$. The horizontal coordinates are the corresponding openings. The maximum error is at a $10 \%$ opening, and the error value is only $6.69 \%$. The minimum error is at a $100 \%$ opening, and the error value is only $0.29 \%$. This shows that the valve has good linear flow characteristics.

\subsubsection{Numerical simulation of the flow field pressure and flow velocity.}

The steady-state field is taken as the initial value of the transient field to accelerate convergence. The computational domain is the whole fluid field. The effect of gravity acceleration on fluid flow is considered. The time step size is set to $0.00025 \mathrm{~s}$, and the number of time steps is set to 8000 . The flow pressure distributions of the flow field at the 2 nd second at openings of $10 \%, 50 \%$ and $100 \%$ are analyzed, as shown in Fig. 5.

According to Fig. 5, the maximum and minimum pressures are 10 and $3 \mathrm{MPa}$, respectively, and are distributed at the inlet and outlet of the valve. At a 10\% opening, the lowest orifices of the inner sleeve are in the flow state. The fluid pressure drops significantly when the fluid flows through the orifices. At this time, the flow rate is relatively low, and the fluid pressure does not change significantly as the fluid flows through the middle sleeve and outer sleeve. At openings of $50 \%$ and $100 \%$, when the fluid passes through the multilayer sleeve, the static pressure of the fluid drops significantly. The total pressure difference of the valve is separated into several small pressure differences, the pressure difference of each stage is greater than the blocked flow pressure difference, and the pressure is greater than the saturated vapor pressure after pressure reduction. This shows that the multilayer sleeve can effectively prevent cavitation and flash. The orifices of the inner sleeve not only control the flow rate well but also have a pressure reducing function.

The flow velocity distribution and the velocity distribution of the vortex core in the internal flow field at openings of $10 \%, 50 \%$ and $100 \%$ are shown in Figs. 6 and 7.

Fig. 6 Velocity distribution of the flow field at different openings.a $10 \%$ opening, b 50\%opening and c $100 \%$ opening

From Fig. 6, the flow velocity increases significantly as the flow area at the multilayer sleeve decreases. The maximum velocity at three openings is distributed at the orifices of the multilayer sleeve. Figure 6(a) shows the flow velocity distribution in the flow field at a $10 \%$ opening. At this time, the maximum velocity is distributed at $151.7 \mathrm{~m} / \mathrm{s}$. The fluid in most areas is stationary in the flow field. At openings of $50 \%$ and $100 \%$, as the throttle area increases, the maximum fluid velocity decreases. However, the fluid in most areas of the flow field is in a flow state and a vortex is easily formed.

In Fig. 7, most vortex cores are distributed around the orifices of the multilayer sleeve. As the opening increases, the number of vortices at the orifices gradually increases, and the vortex core velocity gradually decreases. Some large vortices gradually become small vortices, and some vortices begin to shed, which 
can easily cause vortex-induced vibration in the valve. In addition, the pressure fluctuations become more active. Therefore, the main frequency of the lift coefficient of the vortex shedding process and the main frequency of the pressure fluctuations can be used to evaluate the main frequency of the vortex-induced vibration.

\section{Frequency spectrum analysis of the vortex-induced vibration based on the lift coefficient}

Vortex shedding is a main reason for lateral vortex-induced vibration. Transverse flow-induced vibration is the main form of vortex-induced vibration. When vortex shedding occurs, a drag force forms in the downstream direction, and a lift force forms in the crossflow direction. The average lift force is represented by the lift coefficient, which is dimensionless. The frequency of the peak value of the lift coefficient power spectral density can be considered a parameter for evaluating the main frequency of vortex shedding. The frequency of vortex shedding can be obtained by applying a fast Fourier transform (FFT) to the time-domain information of the lift coefficient obtained through simulation of the transient flow field [15-16]. The lift coefficient expression is:

$$
C_{L}=\frac{F_{L}}{0.5 \rho U^{2} A}
$$

1

where $F_{L}$ is the vortex-induced lift force; $\rho$ is the density of the fluid, $\mathrm{kg} / \mathrm{m}^{3} ; U$ is the flow velocity in the vortex core, $\mathrm{m} / \mathrm{s}$; and $A$ is the cross-sectional area of the vortex.

In the transient flow field, the lift coefficients of the vortices at different openings are monitored, and the corresponding time-domain information is obtained in 2 seconds, as shown in Fig. 8.

(a) (b) (c)

Figure 8 Time domain curves of the lift coefficient in 2 s. a 10\% opening, b $50 \%$ opening and c $100 \%$ opening

According to Fig. 8, the lift coefficient varies from 7.384 to 7.614 at a $10 \%$ opening. The lift coefficient varies from 0.124 to 0.168 at a $50 \%$ opening. The lift coefficient varies from 0.033 to 0.087 at a $100 \%$ opening. The variation range of the lift coefficient at each opening is relatively small, and the lift coefficient decreases as the opening increases.

A fast Fourier transform (FFT) is applied to the time-domain information of the lift coefficient, and the frequency-domain curve of the corresponding lift coefficient is obtained as shown in Fig. 9.

In Fig. 9, the ordinate is the power spectral density of the lift coefficient, and the frequency of the peak value for the lift coefficient power spectral density is the main frequency of vortex shedding. At a $10 \%$ opening, the peak value of the lift coefficient power spectral density is significantly higher than at other 
openings. There are two main frequencies of vortex shedding in the ranges of $0-5 \mathrm{~Hz}$ and $80-140 \mathrm{~Hz}$. At openings of $50 \%$ and $100 \%$, the main frequency of vortex shedding is in the range of $0-40 \mathrm{~Hz}$. The opening of the valve has a significant influence on the main frequency of vortex shedding.

(a) (b) (c)

Figure 9 Frequency spectrum of lift coefficient. a 10\% opening, b 50\% opening and c $100 \%$ opening

This analysis shows that the main frequency of vortex-induced vibration is in the range of $0-140 \mathrm{~Hz}$. At each opening, the fluid flows through the multilayer sleeve, the throttling area decreases sharply, and vortex shedding occurs easily. At small openings, the largest flow velocity occurs at the orifices. The flow velocity changes rapidly. The main frequencies of vortex shedding occur twice, and the distribution range is wide.

\section{Frequency spectrum analysis of the vortex-induced vibration based on the pressure fluctuation}

According to Fig. 7, a large number of vortices are generated around the orifices of the multilayer sleeve in the valve. After vortex shedding, turbulent pressure fluctuations form and are superimposed on each other to produce pulsating shocks. By monitoring the pressure fluctuations at points and surfaces in the flow field, the main frequency of the pressure fluctuation can be regarded as the main frequency of vortexinduced vibration [17-18].

\subsection{Setting of the monitoring points and monitoring surfaces.}

To obtain the time domain information of the pressure fluctuation in the flow field, a fluid-solid coupling surface and points are set, as shown in Fig. 10a and 10b.

(a) (b)

Figure 10 Fluid-solid coupling surface and monitoring points. $\mathbf{a}$ fluid-solid coupling surface and $\mathbf{b}$ monitoring points

Figure 10a shows the fluid-solid coupling surface in the valve. In Fig. 10b, monitoring points P1 P9 are distributed on a symmetrical plane of the fluid channel. Monitoring points S1 S2 and S3 S4 are located near the inner wall of the valve body at the inlet and outlet, respectively.

\subsection{Time domain and frequency domain analysis of the pressure fluctuation.}

To obtain the main frequency of the pressure fluctuation in the flow field, the monitoring points are divided into two categories: monitoring points (P1 P6, S1 S2) in front of the multilayer sleeve and monitoring points (P7 P9, S3 S4) behind the multilayer sleeve. The time domain information of the pressure fluctuations is monitored, as shown in Figs. 11, 12 and 13. 
(a) (b) (c)

(a) (b) (c)

(a) (b) (c)

Figure 13 Time domain curves of pressure fluctuation at $100 \%$ opening. a monitoring points in front of multilayer sleeve, $\mathbf{b}$ monitoring points behind multilayer sleeve and $\mathbf{c}$ fluid solid coupling surface

A fast Fourier transform (FFT) is applied to the time domain information of the pressure fluctuations, and the corresponding frequency domain characteristic curve is obtained, as shown in Figs. 14, 15 and 16.

According to Figs. 14, 15 and 16, the ordinate is the pressure fluctuation power spectrum density. The abscissa is the pressure fluctuation frequency. Therefore, the frequency of the peak value of the pressure fluctuation power spectrum density is the main frequency of vortex-induced vibration. At a $10 \%$ opening, the power spectral densities of the pressure fluctuations do not have an obvious main frequency at monitoring points $\mathrm{P} 1 \sim \mathrm{P} 4$ and $\mathrm{S} 1 \sim \mathrm{S} 2$. The main frequencies of the pressure fluctuations at monitoring points P5 P9 and S3 S4 fluctuate within $20 \mathrm{~Hz}$. At a $50 \%$ opening, the power spectral densities of pressure fluctuations do not have an obvious main frequency at monitoring points P1 P3 and S1 S2. The main frequencies of the pressure fluctuations at monitoring points P4 P6 are within $30 \mathrm{~Hz}$. The main frequencies of the pressure fluctuations at monitoring points P7 P9 and S3 S4 are within $160 \mathrm{~Hz}$. At a $100 \%$ opening, the power spectral densities of the pressure fluctuations do not have an obvious main frequency at monitoring points $\mathrm{P} 1 \sim \mathrm{P} 3$ and S1 S2. The main frequencies of the pressure fluctuations at monitoring points P4 P5 are within $60 \mathrm{~Hz}$. The main frequencies of the pressure fluctuations at monitoring points $\mathrm{P} 6 \sim \mathrm{P} 7$ are within $100 \mathrm{~Hz}$. At monitoring points $\mathrm{P} 8 \sim \mathrm{P} 9$ and S3, the main frequencies of the pressure fluctuations are within $170 \mathrm{~Hz}$. The main frequencies of the pressure fluctuations at monitoring point S4 are within $180 \mathrm{~Hz}$. On the fluid-solid coupling surface, the main frequency of pressure fluctuation is within $20 \mathrm{~Hz}$ for different openings. Combined with the three opening degrees, the main frequency range of the vortex-induced vibration is calculated to be $0 \sim 180 \mathrm{~Hz}$.

(a) (b) (c)

(a) (b) (c)

(a) (b) (c)

Figure 16 Frequency spectrums of pressure fluctuation at $100 \%$ opening. a monitoring points in front of multilayer sleeve, $\mathbf{b}$ monitoring points behind multilayer sleeve and $\mathbf{c}$ fluid solid coupling surface

The pressure fluctuations at the monitoring points in front of the multilayer sleeve do not have obvious main frequencies. At this time, the flow field is relatively stable and approximate to laminar flow. A large number of vortices and vortex shedding form near and behind the multilayer sleeve, and the power spectrum density of the pressure fluctuation fluctuates significantly. Because the pressure fluctuation on 
the fluid-solid coupling surface is the average value of the main frequency and the power spectral density of the pressure fluctuation fluctuates slightly in front of the multilayer sleeve, the monitored information cannot be used to accurately evaluate the main frequency of vortex-induced vibration.

Behind the multilayer sleeve, the pressure fluctuations are superimposed on each other, and the highest main frequency range of the pressure pulsations occurs. Due to the influence of channel shape, the streamlines usually turn back at the inner wall of the valve body and overlap at monitoring point S4. Therefore, the widest main frequency range occurs, and the power spectrum density amplitude of the pressure fluctuation is largest at monitoring point S4. This indicates that the opening and flow channel shape have an effect on the main frequency of vortex-induced vibration.

\section{Thermal-fluid-solid Coupling Modal Analysis}

Under the ANSYS Workbench platform, the fluid module, temperature field module, static module and modal analysis module are combined to calculate the prestress mode (wet mode) of the valve [19-20]. In the fluid module, the pressure and temperature distribution can be obtained by calculating the steadystate flow field of the valve. In the temperature module, the temperature distribution of the valve is obtained by transmitting the fluid temperature to the valve through the coupling surface. In the static module, the fluid pressure is transmitted to the valve through the coupling surface, and the temperature of the valve in the temperature field is imported to the static module. Then, the calculations from the static module are imported to the modal module to complete the modal calculation (wet mode).

In the ANSYS static module, the pressure information and temperature information are loaded into the valve. A fixed constraint is applied to the inlet of the valve, and a displacement constraint is applied to the outlet. The influence of gravitational acceleration is considered. High-order modal frequencies can be considered combinations of several low-order modal frequencies. Therefore, a 1st- to 6th-order modal simulation analysis at 3 openings is carried out. Table 2 shows the material parameters of the main parts of the valve. Table 3 shows the 1 st- to 6 th-order natural frequencies of the valve under the condition of thermal-fluid-solid coupling. 
Table 2

Material parameters of main parts

\begin{tabular}{|c|c|c|c|c|c|}
\hline Parts & Material & $\begin{array}{l}\text { Density } \\
/ \mathrm{kg} / \mathrm{m}^{3}\end{array}$ & $\begin{array}{l}\text { Modulus of } \\
\text { elasticity } \\
\text { /MPa }\end{array}$ & $\begin{array}{l}\text { Poisson's } \\
\text { ratio }\end{array}$ & $\begin{array}{l}\text { Thermal expansion } \\
\text { coefficient } \\
/ \times 10^{-6} /{ }^{\circ} \mathrm{C}\end{array}$ \\
\hline $\begin{array}{l}\text { Valve rod } \\
\text { Valve seat }\end{array}$ & 316SST & 7930 & 179000 & 0.30 & 17.4 \\
\hline $\begin{array}{l}\text { Valve body } \\
\text { Valve bonnet }\end{array}$ & WCB & 7750 & 202000 & 0.30 & 11.55 \\
\hline $\begin{array}{l}\text { Valve plug } \\
\text { Multilayer } \\
\text { sleeve }\end{array}$ & 15CrMo & 7880 & 212000 & 0.28 & 13.37 \\
\hline Pressure cage & & & & & \\
\hline
\end{tabular}

Table 3

Modal frequencies of the valve/ $\mathrm{Hz}$

\begin{tabular}{|lllllll|}
\hline Opening & 1st order & 2nd order & 3rd order & 4th order & 5th order & 6th order \\
\hline $10 \%$ & 257.12 & 821.57 & 914.32 & 962.31 & 1226.1 & 1571.2 \\
\hline $50 \%$ & 256.86 & 817.89 & 914.11 & 962.14 & 1224.3 & 1568.4 \\
\hline $100 \%$ & 255.96 & 812.58 & 908.03 & 951.95 & 1223.3 & 1560.6 \\
\hline
\end{tabular}

In Table 3, the modal frequencies of each order are relatively smaller at a 100\% opening. At each opening, the high-order modal frequencies of the valve are greater. When the valve is at a $100 \%$ opening, the lowest modal frequency of the valve occurs, which is $255.96 \mathrm{~Hz}$.

By comparing the main frequency of vortex shedding with the modal frequency of the valve, there is not an equal or close frequency value at 3 openings, and vortex-induced resonance cannot occur in the valve.

\section{Comparative Analysis Of Monitoring Methods For Vortex-induced Vibration}

By analyzing the lift coefficient power spectral density, the main frequency of vortex-induced vibration in the valve is obtained, which is within $140 \mathrm{~Hz}$. By analyzing the pressure fluctuation power spectral density, the main frequency of vortex-induced vibration in the valve is evaluated, which is within $180 \mathrm{~Hz}$.

The main frequency ranges of vortex-induced vibration at openings of $10 \%, 50 \%$, and $100 \%$ are evaluated using two methods, as shown in Table 4. 
Table 3

The main frequency ranges of vortex-induced vibration $/ \mathrm{Hz}$

\begin{tabular}{|llll|}
\hline Opening & $10 \%$ & $50 \%$ & $100 \%$ \\
\hline Monitoring the lift coefficient & $0-5 ; 80-140$ & $0-40$ & $0-40$ \\
\hline Monitoring the pressure fluctuation & $0-20$ & $0-160$ & $0-180$ \\
\hline
\end{tabular}

At a $10 \%$ opening, the main frequencies of vortex-induced vibration evaluated by monitoring the lift coefficient are in the range of $0-5 \mathrm{~Hz}$ and $80-140 \mathrm{~Hz}$. The main frequency of vortex-induced vibration is within $20 \mathrm{~Hz}$ by monitoring the pressure fluctuation. The frequency of $80-140 \mathrm{~Hz}$ is not captured by monitoring pressure fluctuations, so this method is unreliable at small openings. At this time, the lowest orifices of the inner sleeve are in the flow state. The number of vortices is relatively small. Due to the limitations of the location and number of monitoring points, it is difficult to accurately capture the most active pressure fluctuation. The problems of an insufficient number and inaccurate location of monitoring points can be avoided by monitoring the lift coefficient. Therefore, the main frequency of vortex-induced vibration obtained by monitoring the lift coefficient is more accurate at a small opening. At openings of $50 \%$ and $100 \%$, the main frequencies of vortex-induced vibration are within $40 \mathrm{~Hz}$ by monitoring the lift coefficient. The main frequencies of vortex-induced vibration measured by monitoring the pressure fluctuations are in the range of $0-1180 \mathrm{~Hz}$. The mechanism of monitoring the lift coefficient is to monitor the generation, development and shedding process of the vortex. However, factors such as the superposition effect of the pressure fluctuations after vortex shedding and the flow channel shape are not considered when monitoring the lift coefficient spectrum. At this time, the number of vortices obviously increases, and the effect of the superposition of the pressure fluctuations becomes increasingly significant. In addition, the increased number of vortices increases the likelihood of capturing active pressure fluctuations. Therefore, the main frequency of vortex-induced vibration obtained by monitoring the pressure fluctuation is more accurate at medium and large openings.

\section{Conclusion}

In this paper, vortex-induced vibration characteristics of multistage pressure reducing valves are analyzed using two different methods. By analyzing the lift coefficient power spectral density, the main frequency of vortex-induced vibration in the valve is obtained, which is within $140 \mathrm{~Hz}$. By analyzing the pressure fluctuation power spectral density, the main frequency of vortex-induced vibration in the valve is evaluated, which is within $180 \mathrm{~Hz}$. The lowest modal frequency of the valve is $255.96 \mathrm{~Hz}$. The valve has good vortex-induced vibration characteristics, and vortex-induced resonance does not occur. Monitoring the lift coefficient monitors all vortex shedding processes, but does not consider the superposition of pressure fluctuations after vortex shedding and the influence of flow channel shape. At a small opening, the number of vortices in the flow field is small. The velocity of the flow field varies dramatically. The superposition effect of the pressure fluctuation and the influence of the flow channel shape is not obvious. So monitoring the lift coefficient is more suitable for evaluation of the vortex-induced vibration characteristics of the valve at a small opening. Monitoring the pressure fluctuation is seriously affected 
by the location and number of monitoring points. The selected location and quantity of set monitoring points are very important, and determine the evaluation accuracy. The main frequency of vortex-induced vibration is easier to detect when monitoring points are set near and behind the multilayer sleeve. The method of monitoring the pressure fluctuation on the fluid-solid coupling surface for evaluating the main frequency of vortex-induced vibration of the valve is unreliable. At medium and large openings, the number of vortices in the flow field is larger. the influence of the superposition effect of pressure fluctuations and flow channel shape is more obvious. It is more appropriate to monitor the pressure fluctuation spectrum at medium and large openings. So, a combination of these two methods can more accurately evaluate the vortex-induced vibration characteristics of the valve.

\section{Declarations}

\section{Acknowledgments}

This research were supported by the Fund Project of Liaoning Education Department, China (no. LJKZ0299).

\section{References}

1. Qian, J., Wu, J., Gao, Z et al (2020) Pilot Pipe and Damping Orifice Arrangements Analysis of a PilotControl Globe Valve. J. Fluids Eng 142(10): 101210. https:// doi. org/ 10.1115/1.4047533

2. Mair, M., Bacic, M., and Ireland, P. (2019) On Dynamics of Acoustically Driven Bistable Fluidic Valves. J Fluid Eng 141(6): 061202. https:// doi. org/ 10.1115/1.4041890

3. Qian, J., Zhang, M., Lei, L. et al (2016) Mach number analysis on multi-stage perforated plates in high pressure reducing valve. Energy Convers. Manag 119: 81-90. https:// doi. org/ 10.1016/j.enconman.2016.04.029

4. Li, S., Zhang, W., Wang, W. et al (2020) Simulation for VIV of high pressure drop labyrinth-sleeve combined regulating valves. Vib. Shock 39(03): 95-103.

5. Li, S., Kang, Y., Meng, L. et al (2020) Flow-induced noise of a high pressure drop control valve. Vib. Shock 39(14): 116-121.

6. Wang, H., Kong, X., Liu, H. (2018) Vibration characteristics analysis for a tri-eccentric butterfly valve. J. Shock. Vib. 37(05): 202-206+212.

7. Wang, H., Liu, H., Kong X. (2019) Vortex Shedding of the Tail in the Flow Passing through a Trieccentric Butterfly Disc. Fluid Mech 47(2): 23-29.

8. Kang Z, Zhang. L., Ni, W. (2017) Vortex-induced vibration of a cylinder considering hysteretic effect. J. Vib. Shock. 36(24): 220-229.

9. Gao Y, Yang J., Zou L., Zong Z. (2018) Effects of surface roughness on vortex induced vibration features of a circular cylinder. J. Vib. Shock. 37(9): 37-43. 
10. Chizfahm, A., Yazdi, E. A., et al (2018) Dynamic modeling of vortex induced vibration wind turbines. Renew. Energ., 121: 632-643. https:// doi. org/ 10.1016/j.renene.2018.01.038

11. Youn, C., Asano, S., Kawashima. K. et al (2008) Flow characteristics of pressure reducing valve with radial slit structure for low noise. J Vis 11(4): 357-364.

12. Jin, Z., Qiu, C., Jiang, C., et al (2020) Effect of valve core shapes on cavitation flow through a sleeve regulating valve. J Zhejiang Univer-SCIENCE A 21(21): 1-14.

13. Xu, H., Guang, Z. M., et al (2011) Hydrodynamic characterization and optimization of Contra-push check valve by numerical simulation. Ann Nucl Energy 38(6): 1427-1437.

14. Chen, J., Yuan, C., Guo, Q., et al (2017) Dynamic flow field analysis of the prestage of jet pipe servo valve. Chin J Sci Instrum 38(07): 1731-1737.

15. Cui Z, Zhao. C., Wei Y. Y. (2020) Experimental Research on the Effects of Wear-ring Clearance on the Performance and Vibration Characteristics of Centrifugal Pumps. Fluid Mach 48(1): 1-6.

16. Jiao, H., Wu, G. X. (2021) Effect of Reynolds number on amplitude branches of vortex-induced vibration of a cylinder. J Fluids Struct 105: 103323. https:// doi. org/ 10.1016/J.JFLUIDSTRUCTS.2021.103323.

17. Li, S., Shen, H., Zhang, W. (2020) Cavitation characteristics and pressure pulsation response of control valve under typical opening. J. Huazhong Univ. Sci. Tech. 48(12): 1-8.

18. Binama, M., Kan, K., Chen, H.-X. et al (2021) Flow instability transferability characteristics within a reversible pump turbine (RPT) under large guide vane opening (GVO). Renew Energ 179: 285-307. https:// doi. org/ 10.1016/ J. RENENE .2021.07.039

19. Hou, H., Rui, T., Deng, R., et al (2021) Modal analysis of central impression cylinder based on fluidsolid coupling method. J Low Freq Noise V A 40(2): 772-783.

20. Li, S., Zhu, L., Wang, W., Xiao, K. et al (2019) Analysis of thermal-fluid-structure coupling and resonance forecast for link butterfly valve under small opening. J Shanghai Jiaotong Univer 24(3): 341-350.

\section{Figures}




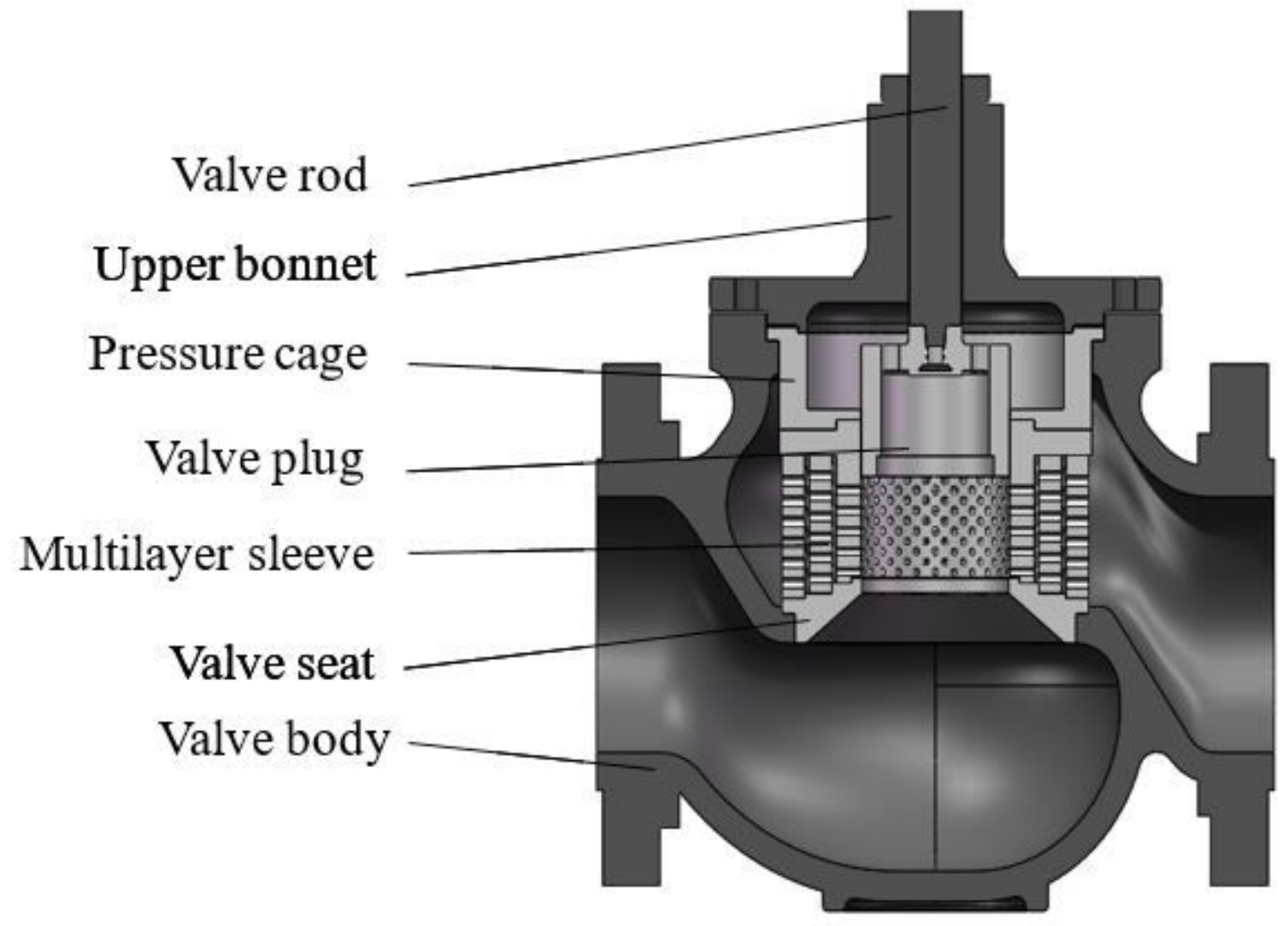

Figure 1

The structure of multistage pressure reducing valve

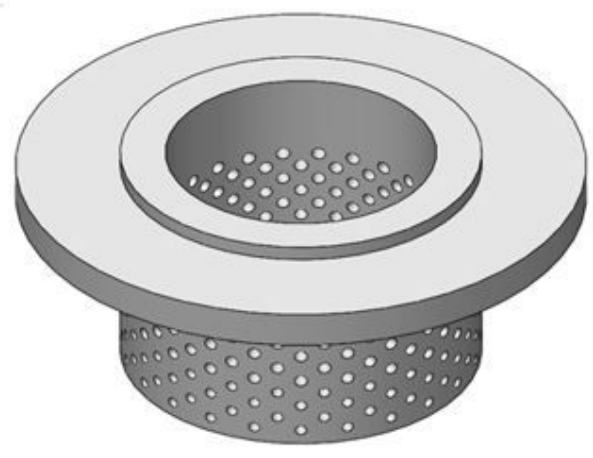

(a)

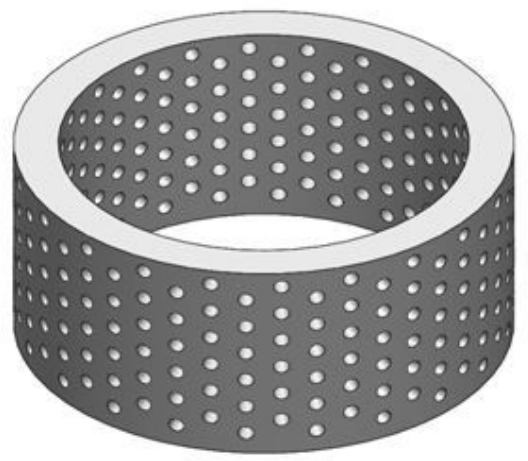

(b)

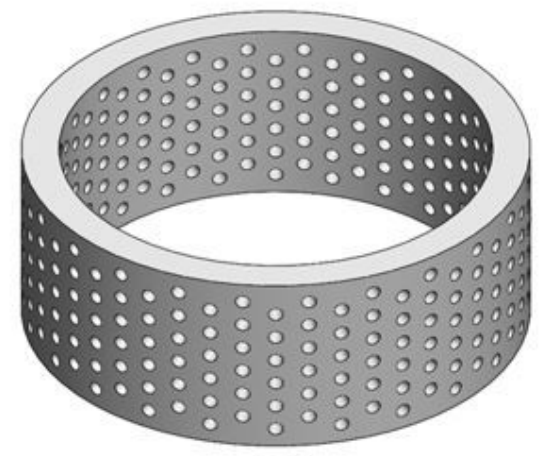

(c)

Figure 2

The multilayer sleeve. a inner sleeve, b middle sleeve and c outer sleeve 


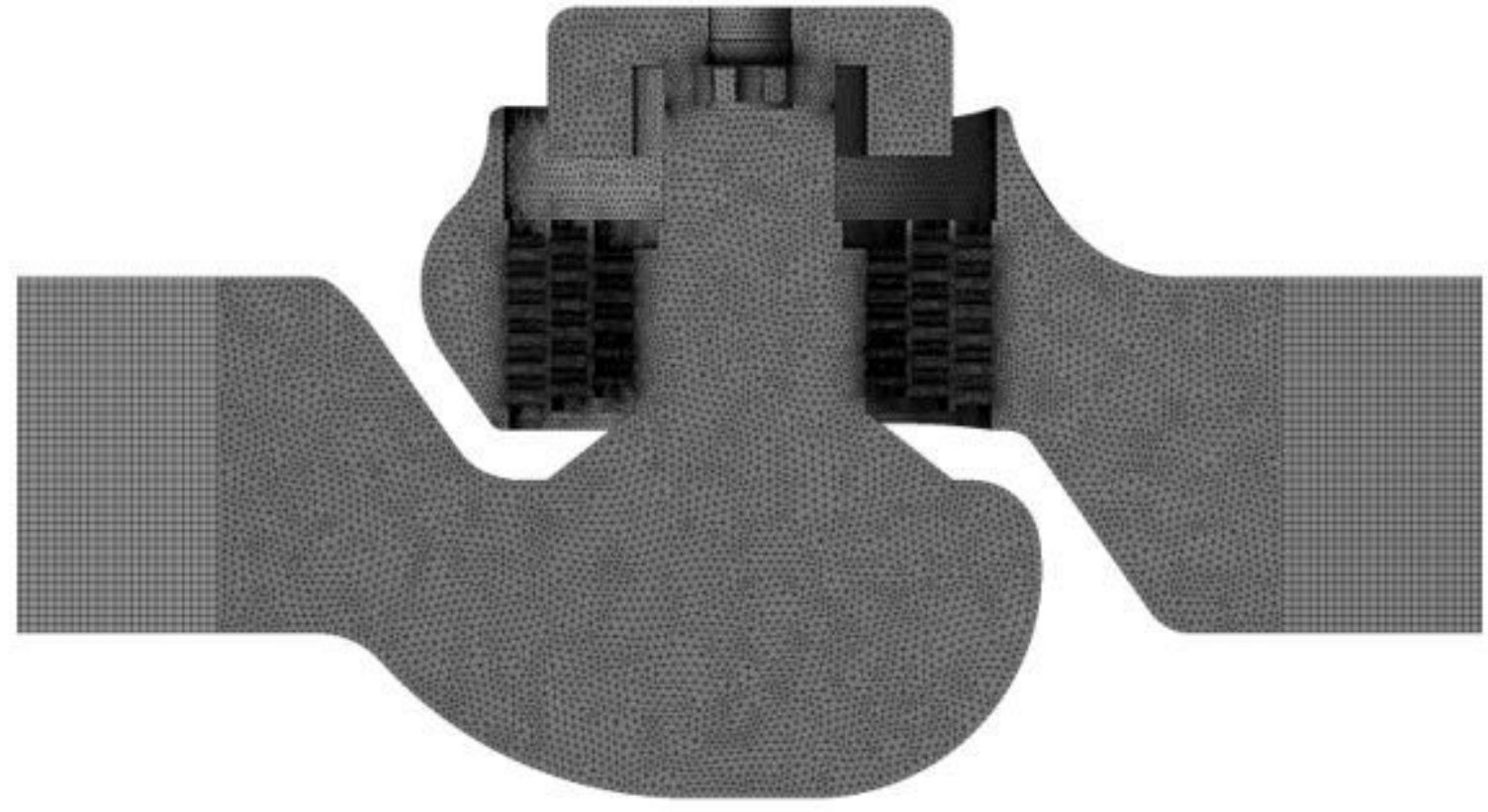

Figure 3

Fluid mesh model of the multistage pressure reducing valve

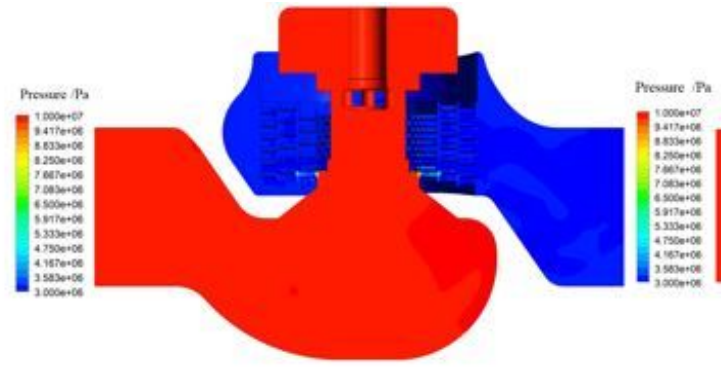

(a)

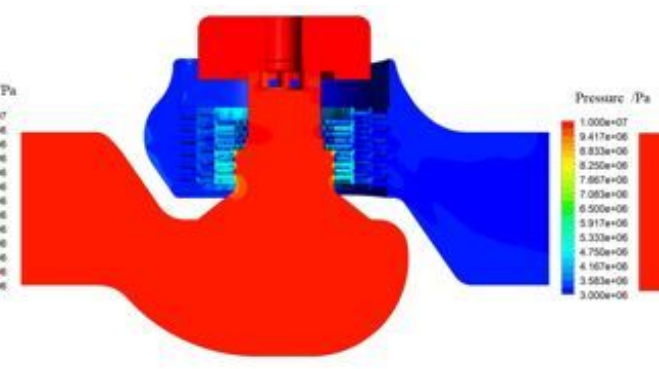

(b)

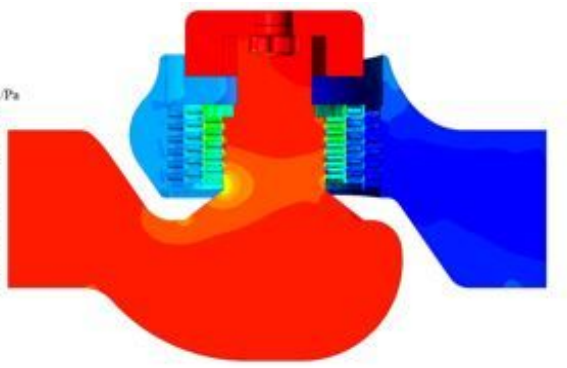

(c)

\section{Figure 4}

Pressure distribution of the flow field at different openings. a 10\% opening, b $50 \%$ opening and c $100 \%$ opening

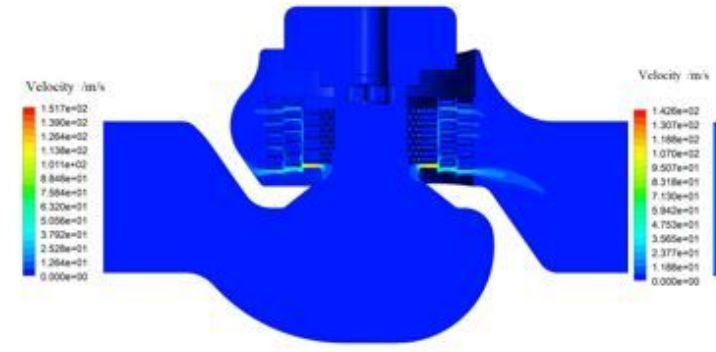

(a)

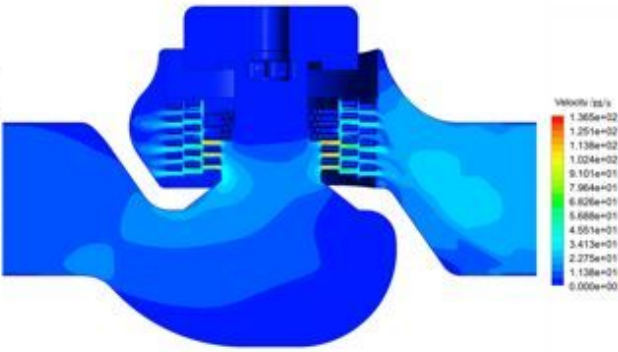

(b)

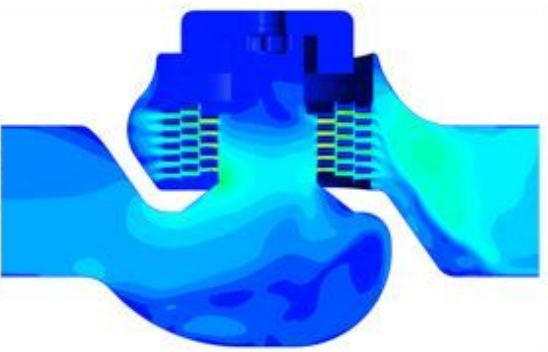

(c)

Figure 5 
Velocity distribution of the flow field at different openings. a $10 \%$ opening, b $50 \%$ opening and c $100 \%$ opening

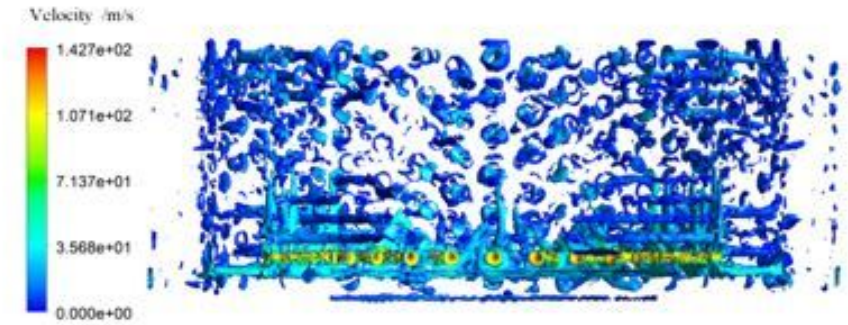

(a)

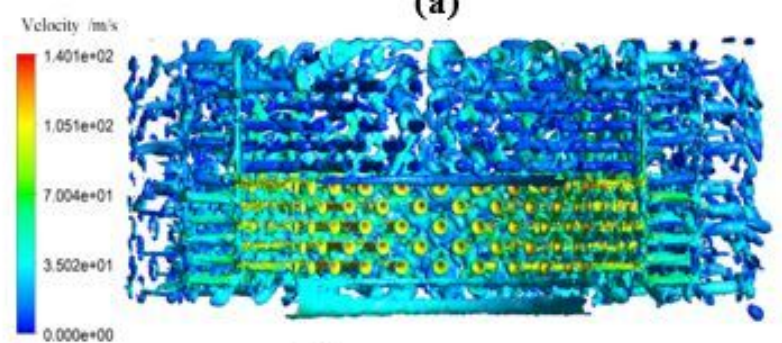

(b)

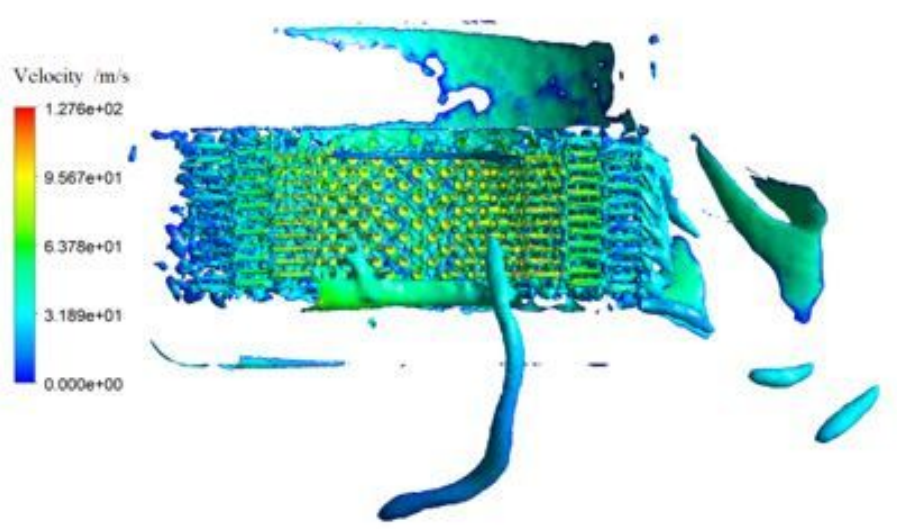

(c)

Figure 6

Velocity distribution of the vortex core at different openings. a $10 \%$ opening, b $50 \%$ opening and c $100 \%$ opening

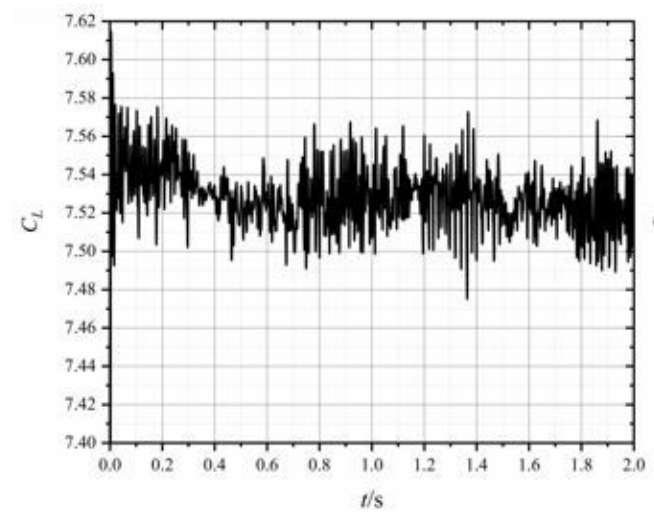

(a)

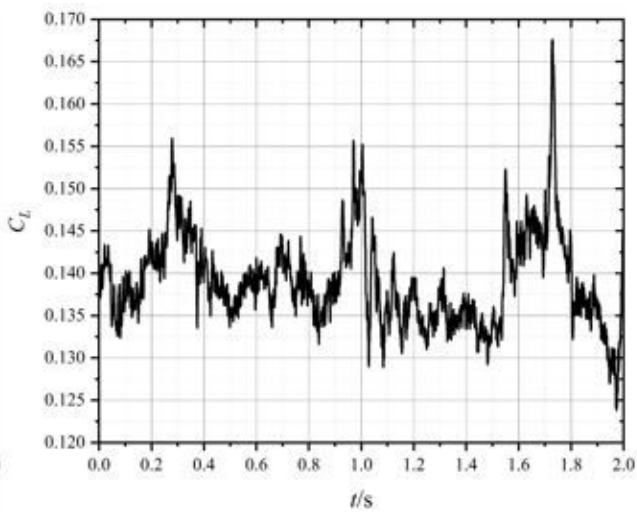

(b)

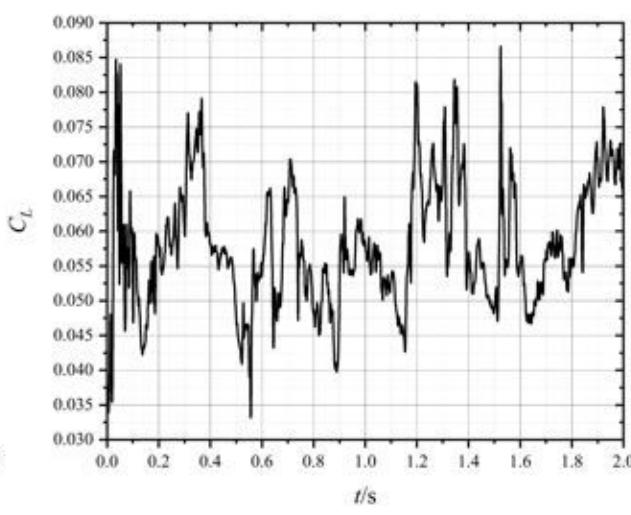

(c)

Figure 7

Time domain curves of the lift coefficient in 2 s. a $10 \%$ opening, b $50 \%$ opening and c $100 \%$ opening 


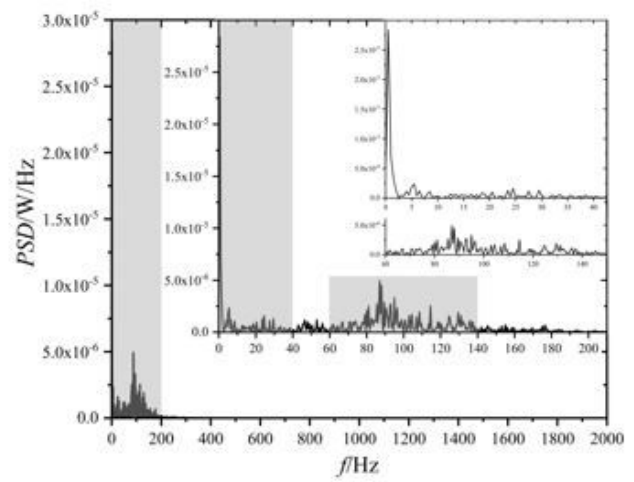

(a)

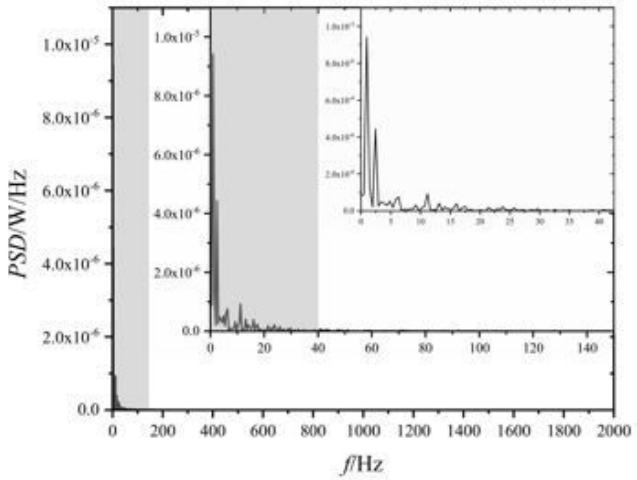

(b)

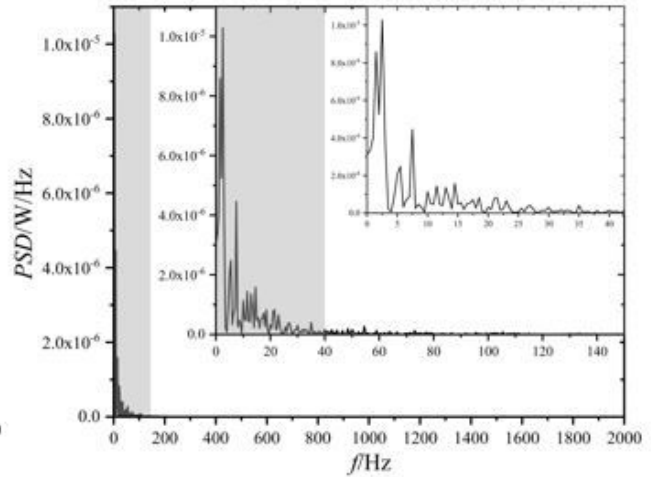

(c)

\section{Figure 8}

Frequency spectrum of lift coefficient. a 10\% opening, b 50\% opening and c 100\% opening

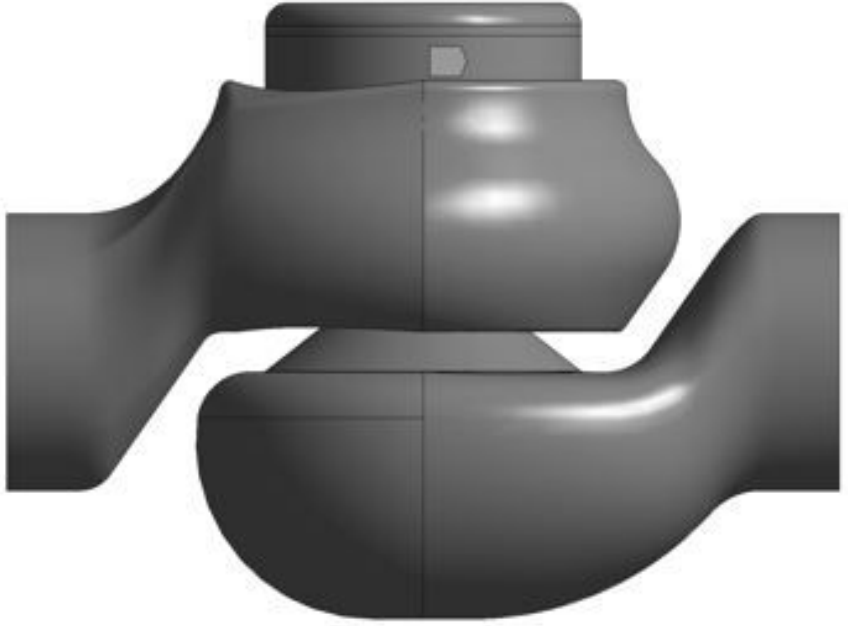

(a)

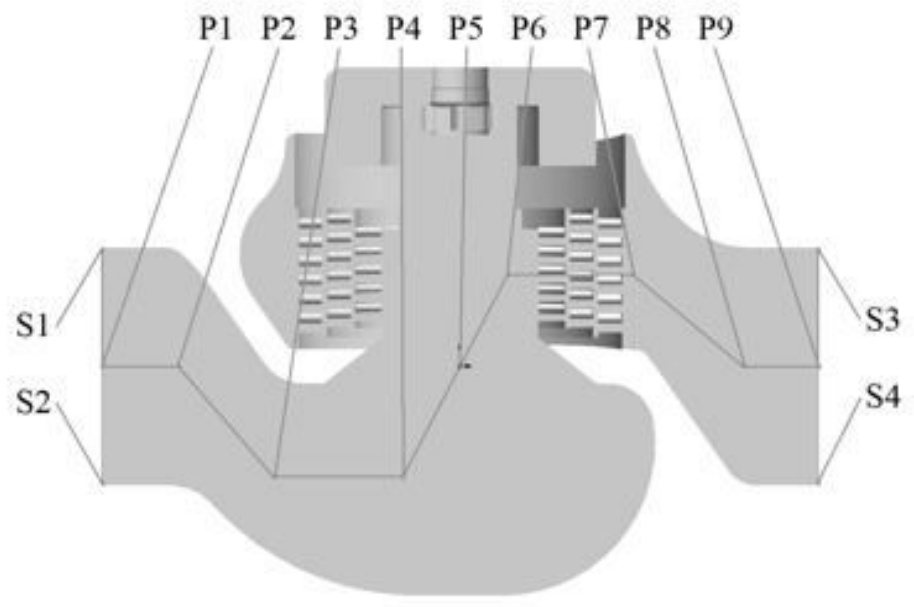

(b)

\section{Figure 9}

Fluid-solid coupling surface and monitoring points. a fluid-solid coupling surface and $b$ monitoring points

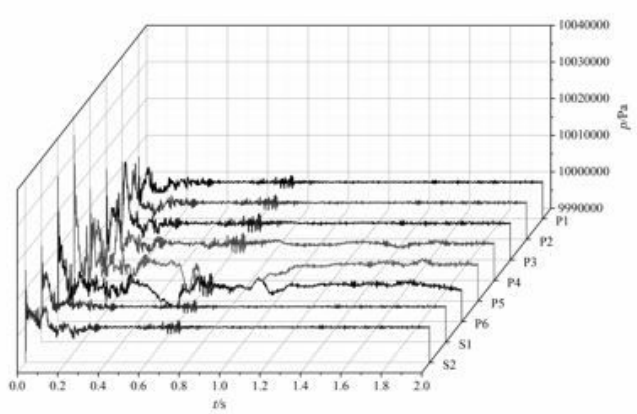

(a)

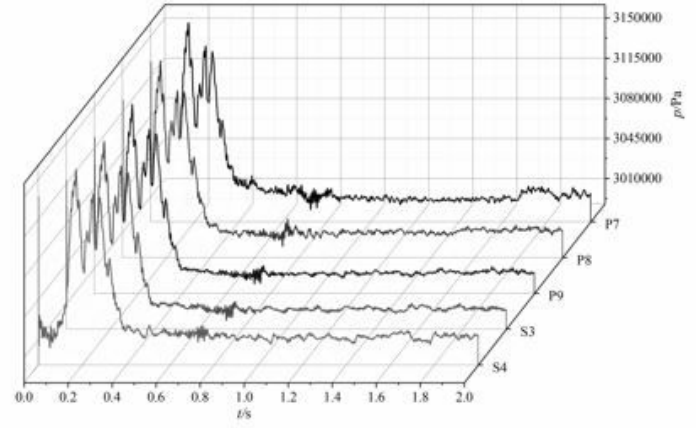

(b)

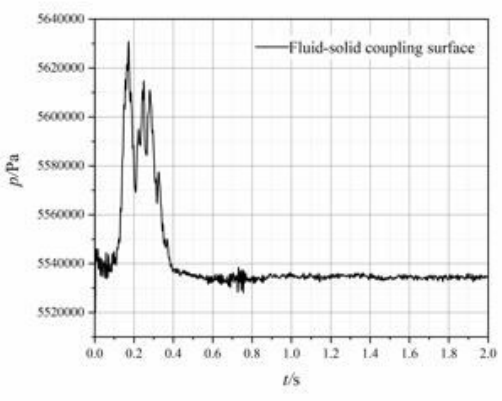

(c) 
Time domain curves of pressure fluctuation at $10 \%$ opening. a monitoring points in front of multilayer sleeve, $b$ monitoring points behind multilayer sleeve and $c$ fluid solid coupling surface

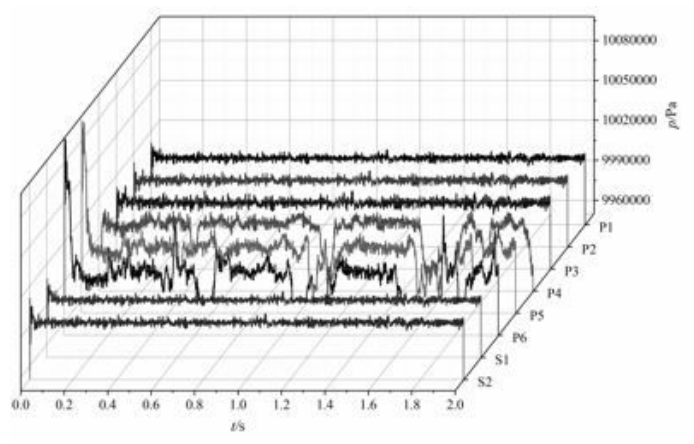

(a)

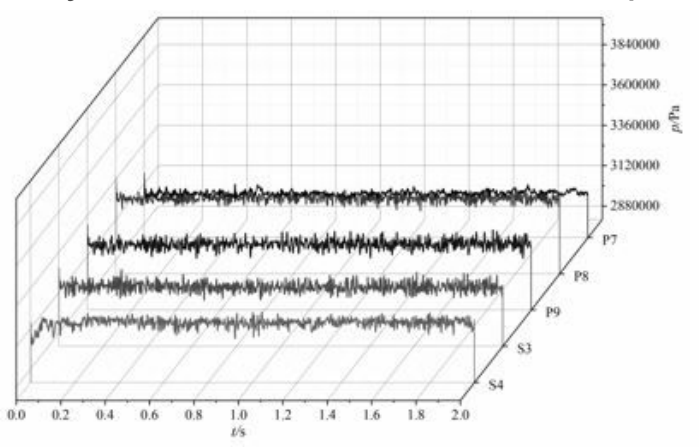

(b)

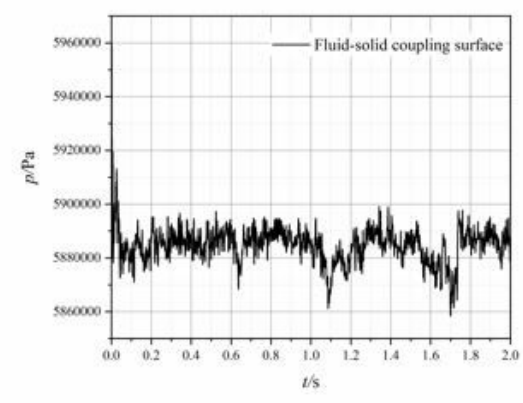

(c)

\section{Figure 11}

Time domain curves of pressure fluctuation at $50 \%$ opening. a monitoring points in front of multilayer sleeve, $b$ monitoring points behind multilayer sleeve and $c$ fluid solid coupling surface

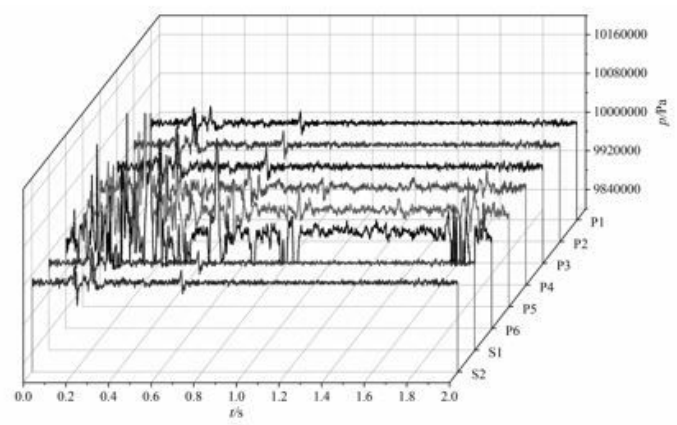

(a)

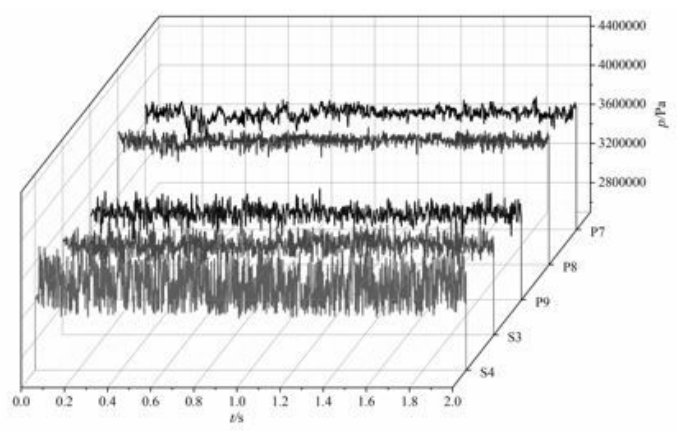

(b)

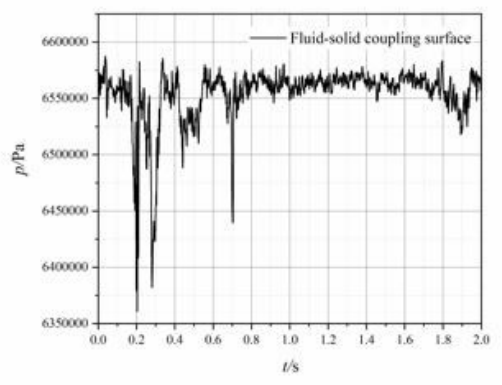

(c)

\section{Figure 12}

Time domain curves of pressure fluctuation at $100 \%$ opening. a monitoring points in front of multilayer sleeve, $b$ monitoring points behind multilayer sleeve and $c$ fluid solid coupling surface

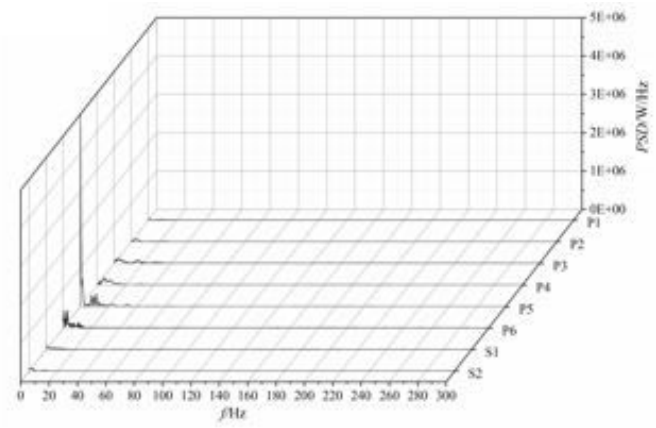

(a)

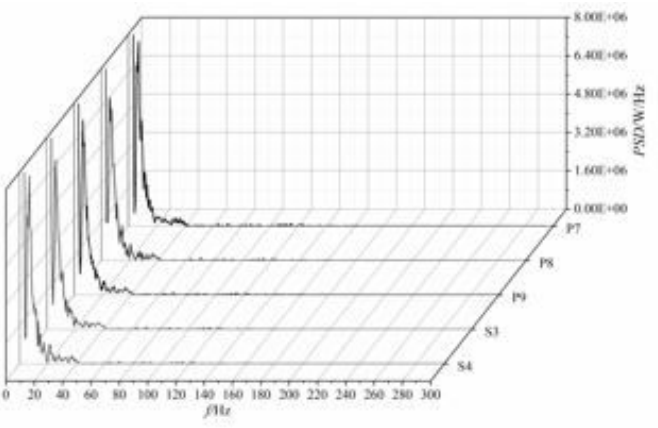

(b)

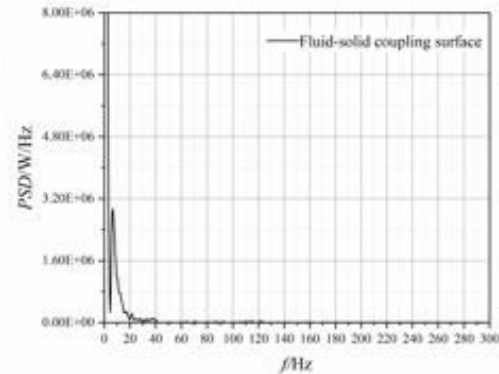

(c)

Figure 13 
Frequency spectrums of pressure fluctuation at $10 \%$ opening. a monitoring points in front of multilayer sleeve, $b$ monitoring points behind multilayer sleeve and $c$ fluid solid coupling surface

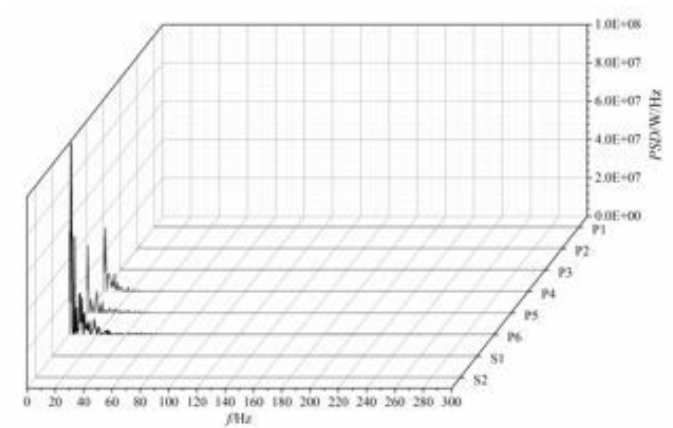

(a)

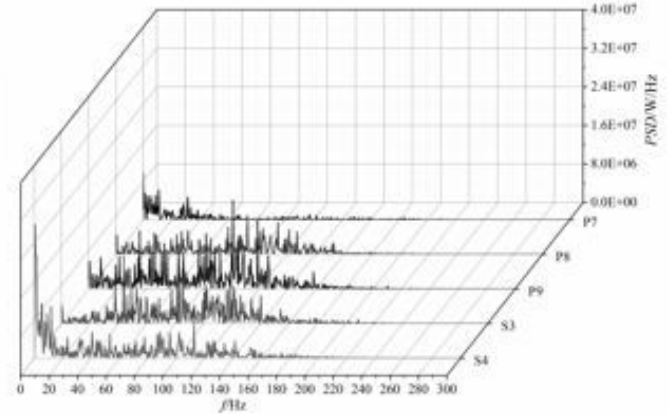

(b)

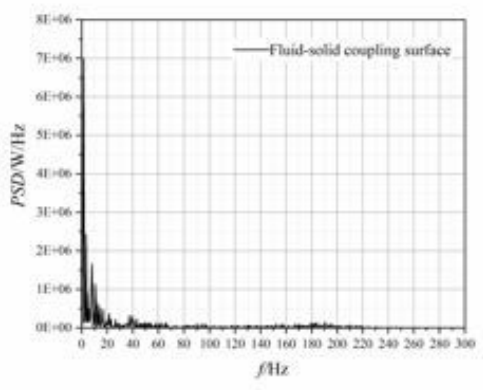

(c)

\section{Figure 14}

Frequency spectrums of pressure fluctuation at $50 \%$ opening. a monitoring points in front of multilayer sleeve, $b$ monitoring points behind multilayer sleeve and $c$ fluid solid coupling surface

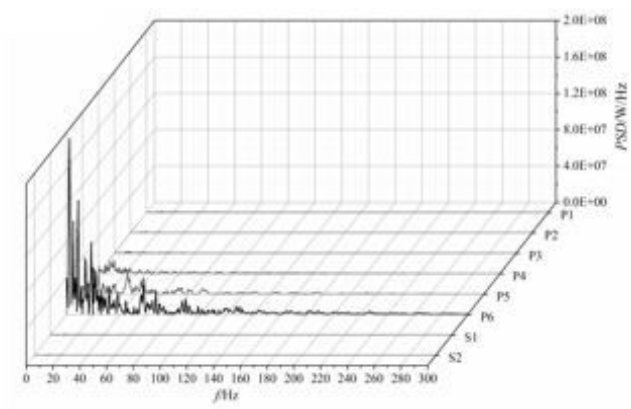

(a)

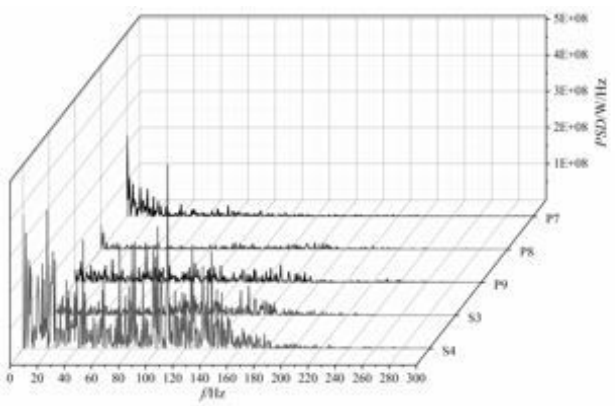

(b)

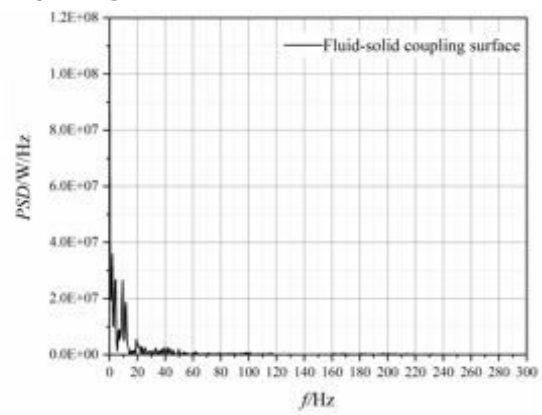

(c)

Figure 15

Frequency spectrums of pressure fluctuation at $100 \%$ opening. a monitoring points in front of multilayer sleeve, $b$ monitoring points behind multilayer sleeve and $c$ fluid solid coupling surface 